\title{
Environmental Variation, Stochastic Extinction, and Competitive Coexistence
}

Peter B. Adler ${ }^{1, *}$ and John M. Drake $e^{2, \dagger}$

1. Department of Wildland Resources and the Ecology Center, Utah State University, Logan, Utah 84322;

2. Odum School of Ecology, University of Georgia, Athens, Georgia 30602

Submitted November 25, 2007; Accepted May 16, 2008;

Electronically published September 24, 2008

ABSTRACT: Understanding how environmental fluctuations affect population persistence is essential for predicting the ecological impacts of expected future increases in climate variability. However, two bodies of theory make opposite predictions about the effect of environmental variation on persistence. Single-species theory, common in conservation biology and population viability analyses, suggests that environmental variation increases the risk of stochastic extinction. By contrast, coexistence theory has shown that environmental variation can buffer inferior competitors against competitive exclusion through a storage effect. We reconcile these two perspectives by showing that in the presence of demographic stochasticity, environmental variation can increase the chance of extinction while simultaneously stabilizing coexistence. Our stochastic simulations of a two-species storage effect model reveal a unimodal relationship between environmental variation and coexistence time, implying maximum coexistence at intermediate levels of environmental variation. The unimodal pattern reflects the fact that the stabilizing influence of the storage effect accumulates rapidly at low levels of environmental variation, whereas the risk of extinction due to the combined effects of environmental variation and demographic stochasticity increases most rapidly at higher levels of variation. Future increases in environmental variation could either increase or decrease an inferior competitor's expected persistence time, depending on the distance between the present level of environmental variation and the optimal level anticipated by this theory.

Keywords: climate variability, competition, demographic stochasticity, plant communities, population dynamics, simulation.

* Corresponding author; e-mail: peter.adler@usu.edu.

† E-mail: jdrake@uga.edu.

Am. Nat. 2008. Vol. 172, pp. E186-E195. (c) 2008 by The University of Chicago. 0003-0147/2008/17205-50077\$15.00. All rights reserved. DOI: $10.1086 / 591678$
The great difficulty in the opportunistic hypothesis is that since, if many species are present in a really variable environment which is controlling their competition, chance extinction is likely to be an important aspect of the process. (Hutchinson 1961, pp. 141-142)

Temporal variation is a ubiquitous feature of ecosystems, and ecologists have long been interested in how the magnitude of variation structures natural populations and communities (Andrewartha and Birch 1954; Lewontin and Cohen 1969). Exogenous environmental variation has been shown to affect all levels of biological organization, from the evolution of individual traits (Cohen 1966; Venable and Brown 1988) to the stability of ecosystem processes (Tilman and Downing 1994; Bai et al. 2004). This basic research must now provide the foundation for understanding the ecological impacts of expected increases in climate variability. Global circulation models consistently forecast increases in the frequency of extreme events such as severe storms and droughts (Karl and Trenberth 2003; Salinger 2005). As precipitation and temperature depart from their historical ranges of variability, theory will be crucial for predicting how natural populations will respond.

Unfortunately, such predictions are complicated by disagreement between two bodies of ecological theory. The single-species models used in population viability analysis, an important tool in conservation biology, suggest that environmental variation typically increases the risk of stochastic extinction due to geometric averaging of population growth rates over time and high spatial synchrony of species with restricted ranges (Boyce 1992; Menges 2000; but see Drake 2005; Boyce et al. 2006). By contrast, coexistence theory has shown that environmental variation can buffer rare species against extinction: temporary conditions resulting in high individual fitness can allow inferior competitors to escape competitive exclusion (Chesson and Warner 1981; Chesson and Huntly 1989). Empirical tests in natural systems indicate that this coexistence mechanism may be important in communities of zooplankton (Cáceres 1997), annual plants (Pake and 
Venable 1995; Adondakis and Venable 2004; Facelli et al. 2005), and prairie grasses (Adler et al. 2006).

As previous authors have noted (Higgins et al. 2000; Levine and Rees 2004), an important difference between these two perspectives is the focus on competitive interactions in coexistence models. But these two disciplines also have addressed subtly different questions. Singlespecies conservation studies typically use stochastic simulations to evaluate mean time to extinction in models that include a quasiextinction threshold or formally incorporate demographic stochasticity (reviewed in Boyce 1992; Menges 2000), whereas coexistence theory uses deterministic, analytical models without extinction thresholds or demographic stochasticity to evaluate whether coexistence is stable in the long term (e.g., Chesson 1990).

Here we aim to reconcile these perspectives by studying the combined effects of environmental variation and demographic stochasticity on mean coexistence times in a modified version of Chesson and Huntly's (1989) twospecies annual plant model. Our approach recognizes that environmental variation can increase the probability of extinction while simultaneously stabilizing coexistence by buffering inferior competitors against competitive exclusion. To understand the combined effect of these two forces on the duration of coexistence, we first study them individually. We show that the probability of stochastic extinction in this model is low at low levels of environmental variation but then increases quickly at higher levels of variation. In contrast, the stabilizing effects of environmental variation accumulate most rapidly at low levels of environmental variation. As a result, in simulations of the two-species model with demographic stochasticity, we find a unimodal relationship between environmental variation and coexistence times, implying that coexistence is maximized at an intermediate level of environmental variation. We explore how the location of this optimum depends on the degree of competitive asymmetry and the correlation in species' responses to the environment.

\section{Methods}

While the effect of environmental variation on extinction in single-species systems has been studied for many population models (Alvarez 2001), research on coexistence mediated by temporal variability is based on a limited set of competition models (Chesson and Warner 1981; Chesson and Huntly 1989; Chesson 2000). To facilitate our comparison of these distinct perspectives, we first demonstrate the single-species approach by using a one-species special case of Chesson and Huntly's (1989) competition model.

\section{Extinction in a Single-Species System}

Changes in the abundance of seeds over time are set deterministically (following Chesson and Huntly 1989) by the nonlinear difference equation

$$
\hat{N}_{t+1}=s\left[1-g\left(E_{t}\right)\right] N_{t}+\frac{\lambda g\left(E_{t}\right) N_{t}}{1+\alpha g\left(E_{t}\right) N_{t}},
$$

where $N_{t}$ is the abundance of seeds at time $t, s$ is the survival of seeds in the seed bank, $\lambda$ is the per capita fecundity, $\alpha$ is the intraspecific competition coefficient, and $g$ is the germination rate. This model allows populations to decline arbitrarily close to, but not reach, extinction. To incorporate demographic stochasticity, we draw the realized $N_{t+1}$ density of seeds at time $t+1$ from a Poisson distribution with expectation $\hat{N}_{t+1}$, that is,

$$
N_{t+1} \sim \operatorname{Pois}\left(\hat{N}_{t+1}\right)
$$

Adding demographic stochasticity means that actual seed densities take integer values and that extinction $(N=0)$ is possible.

We simulated annual germination rates by drawing a sequence of independent and identically distributed random variates, $E$, from a continuous normal distribution with mean 0 and variance $\sigma_{E}^{2}$, and then we used the inverse logit transformation to convert $E$ to germination rates in the $0-1$ range:

$$
g(E)=\frac{e^{E}}{1+e^{E}}
$$

As the variance of $E, \sigma_{E}^{2}$, increases, extreme values for germination occur more frequently (fig. 1). We conducted our simulations at values of $\sigma_{E}^{2}$ of $0,0.4,1,2.5,5,7.5$, and 10.

We used simulations to estimate the probability that the population will reach extinction within short time periods, given a specified variance for $E$. Simulations were initialized at low population density $\left(N_{0}=2\right.$, where $N_{0}$ is the realized density and not the mean of the Poisson process), iterated for 100 time steps, and replicated 10,000 times. We did not follow each run to extinction because the computing time would be prohibitive. The probability of extinction was estimated as the fraction of runs in which density fell to 0 . As a baseline for comparison, we set $s=0.5$ and $\alpha=1$. We repeated the simulations for values of $\lambda$ of $10,15,25$, and 100 to represent a range from small, slow-growing populations to larger, fast-growing populations. 

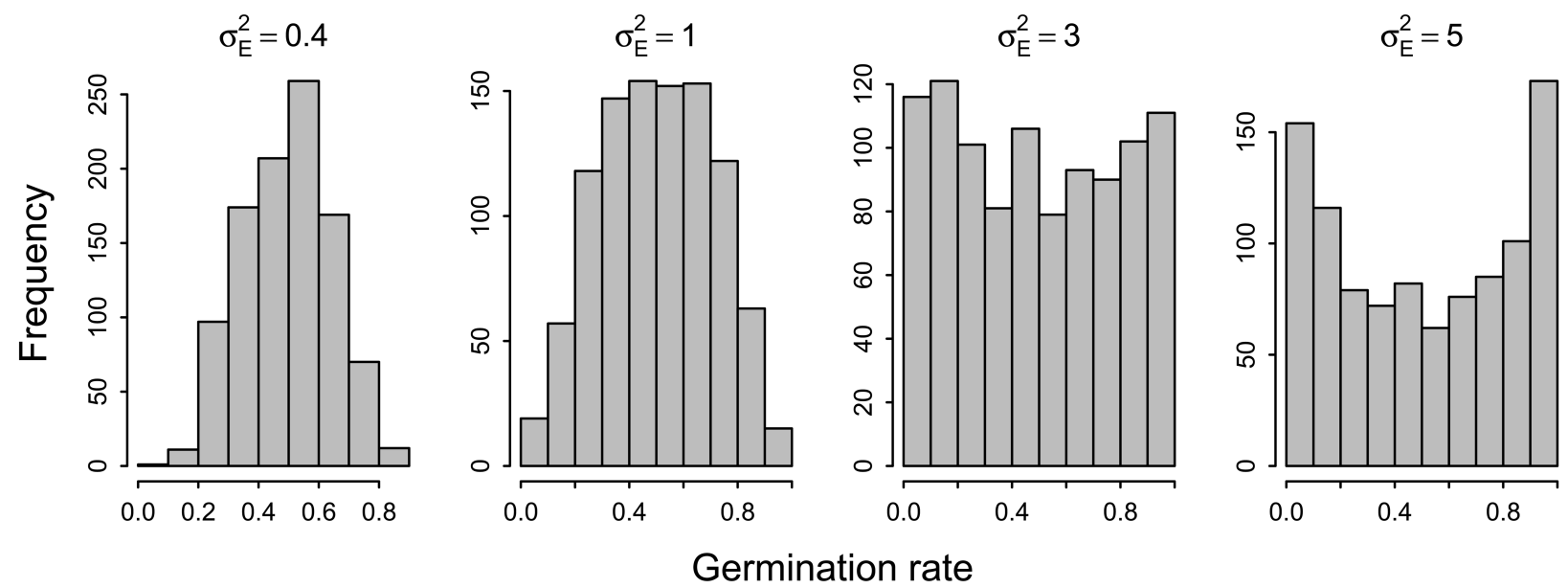

Germination rate

Figure 1: Distribution of germination rates as a function of variation in the underlying normally distributed environment, $\sigma_{E}^{2}$.

\section{The Stabilizing Effect of Environmental Variability}

To demonstrate that temporal variability can allow an inferior competitor to persist even if it would be excluded in a constant environment, Chesson and Huntly (1989) analyzed the following two-species model:

$$
\begin{aligned}
& N_{1, t+1}=s_{1}\left[1-g_{1}\left(E_{t}\right)\right] N_{1, t}+\frac{\lambda_{1} g_{1}\left(E_{t}\right) N_{1, t}}{1+\alpha_{11} g_{1}\left(E_{t}\right) N_{1, t}+\alpha_{12} g_{2}\left(E_{t}\right) N_{2, t}}, \\
& N_{2, t+1}=s_{2}\left[1-g_{2}\left(E_{t}\right)\right] N_{2, t}+\frac{\lambda_{2} g_{2}\left(E_{t}\right) N_{2, t}}{1+\alpha_{21} g_{1}\left(E_{t}\right) N_{1, t}+\alpha_{22} g_{2}\left(E_{t}\right) N_{2, t}} .
\end{aligned}
$$

The variables are the same as in the single-species model, with subscripts denoting species 1 and 2 and $\alpha_{i j}$ representing the per capita competitive effect of species $j$ on species $i$. Assuming that all $\alpha_{i j}=1$ and if both species respond identically to the environment, the outcome of competition is given by $\lambda_{i}$ : the species with the lower fecundity will be excluded. Note that this model does not include demographic stochasticity and that seed abundances can be any real number $>0$.

When species respond differently to a varying environment, it is possible for the inferior to persist (Chesson and Huntly 1989). Environmental variation has a stabilizing effect on coexistence when the model satisfies three conditions (Chesson and Warner 1981; Warner and Chesson 1985; Chesson and Huntly 1989; Chesson 1990). First, the organisms must have some mechanism for persisting during unfavorable periods, such as a seed bank, quiescence, or diapause. This condition, which gives the storage effect its name, buffers population growth; without it, populations would become extinct after a brief unfavorable pe- riod and environmental variation could never promote coexistence. As long as $s>0$, our plant model meets this condition. Second, species must respond differently to environmental variation, making it possible for one species to experience a relatively good year while its competitor is experiencing a relatively bad year. This condition is satisfied in the annual plant models as long as values of $g$ are not perfectly correlated. Third, the effects of competition on a species must be more strongly negative in a year that is good for that species than in an unfavorable year. Given the second condition, which tends to partition intraspecific and interspecific competition into different years, this condition ensures that intraspecific competition will be stronger than interspecific competition, the basic requirement for stabilized coexistence (Chesson 2000). If we hold all $\alpha_{i j}=1$, this condition is also met by our model.

We used an invasibility criterion to quantify the degree to which environmental variation stabilizes the persistence of the inferior species. Any species that increases from low abundance in the presence of competitors at their equilibrium abundances can coexist stably (Chesson 2000). We began our simulations by initializing the inferior species at an abundance of one individual and the superior species at its single-species equilibrium. We then drew the two species' germination rates before projecting population abundance at the next time step in order to calculate the realized population growth rate. We repeated this projection 10,000 times for each parameter combination, drawing a new pair of germination rates for each iteration.

For the two-species case, when germination rates are identical, it is impossible for environmental variation to stabilize coexistence (Chesson and Huntly 1989). The 
(A)

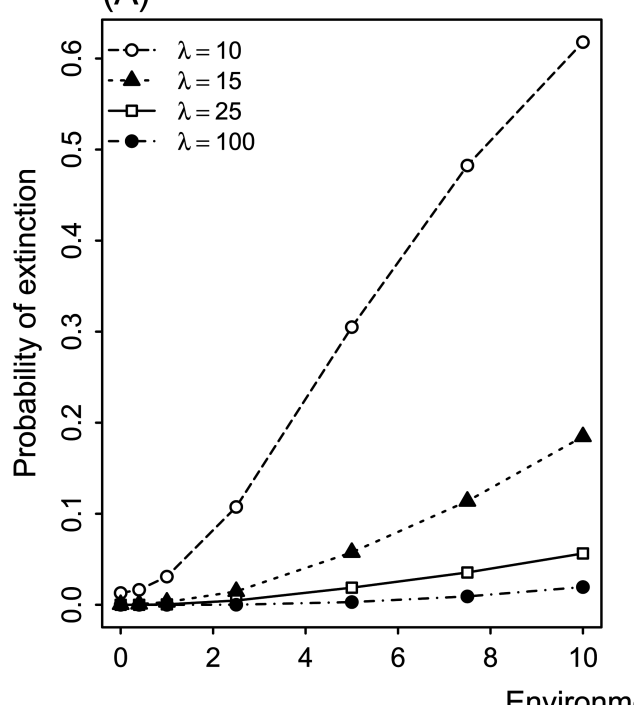

(B)

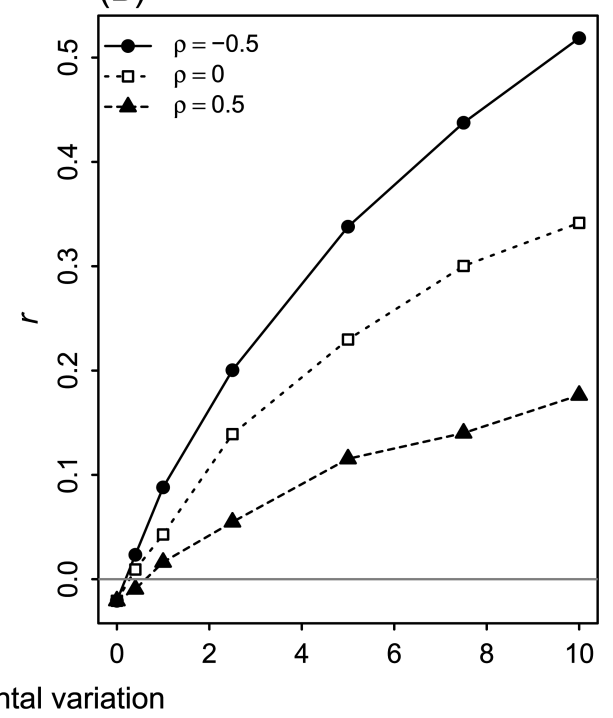

Figure 2: Contrasting results about the effect of environmental variation on extinctions and long-term persistence. $A$, Conservation biology approach. A single-species system initialized at low abundance was simulated to determine the probability of extinction within 100 time steps due to the combined effects of demographic and environmental stochasticity. Extinction risk increases slowly at low levels of environmental variation across a range of values of $\lambda$. B, Theoretical coexistence approach. A two-species system with no demographic stochasticity was simulated to determine the inferior competitor's average log low-density growth rate, $r$, in the presence of an established competitor. At 0 or low levels of environmental variation, $r$ is negative, and coexistence is impossible. As environmental variation increases, $r$ increases above 0 , meaning that the potential for the inferior to persist increases. For different values of the correlation in the two species' germination rates, $\rho, r$ increases most rapidly at low levels of environmental variation.

lower (more negative) the correlation in germination rates, the stronger the potential for the inferior to persist. Thus, the correlation between the two species' germination rates, $\rho$, is an important parameter. For $\rho<1$, we generated sequences of correlated random numbers by drawing from multivariate normal distributions with means of 0 and a variance-covariance matrix of

$$
\left[\begin{array}{cc}
\sigma_{E}^{2} & \rho \sigma_{E}^{2} \\
\rho \sigma_{E}^{2} & \sigma_{E}^{2}
\end{array}\right] .
$$

We converted these sequences of random variates to germination rates using the inverse logit transform, as in the single-species case (eq. [3]).

For all runs, we set $s=0.5$, all $\alpha_{i j}=1, \lambda_{1}=102$, and $\lambda_{2}=98$ (the inferior). We repeated the simulations for seven values of $\sigma_{E}^{2}(0,0.4,1,2.5,5,7.5$, and 10$)$ and three values of $\rho(0.5,0$, and -0.5$)$. The long-term low-density growth rate of the inferior at each parameter combination was calculated as the mean of its log growth rates (Lewontin and Cohen 1969) over the 10,000 projections. We focused on the mean low-density growth rate in the absence of demographic stochasticity rather than the outcome of individual simulations in order to isolate the stabilizing effect of environmental variation.

\section{Simulations of Coexistence Time}

We considered the combined effects of stochastic extinction and stabilization via the storage effect by analyzing the duration of coexistence in a version of the two-species model modified to include demographic stochasticity. We incorporated demographic stochasticity as in the onespecies case, drawing the actual seed density at each time step for species $i$ from a Poisson with a mean equal to the expected seed abundance predicted by the two-species model (eq. [4]):

$$
N_{i, t+1} \sim \operatorname{Pois}\left(\hat{N}_{i, t+1}\right) .
$$

We examined the effect of the following three parameters on coexistence times: (1) the magnitude of environmental variance, $\sigma_{E}^{2}$; (2) the correlation in species' responses to the environment, $\rho$; and (3) the degree of competitive asymmetry (the ratio $\lambda_{1} / \lambda_{2}$ ). Our experiments crossed five levels of $\sigma_{E}^{2}(0,0.4,1,3$, and 5), four levels of $\rho(1,0.5,0$, and -0.5$)$, and three levels of competitive asymmetry $\left(\lambda_{1}=101\right.$ and $\lambda_{2}=99, \lambda_{1}=102$ and $\lambda_{2}=98$, and $\lambda_{1}=105$ and $\lambda_{2}=95$ ). As before, $s$ was set to 0.5 , and all competition coefficients were set to 1 . We performed 5,000 runs of each parameter combination. 

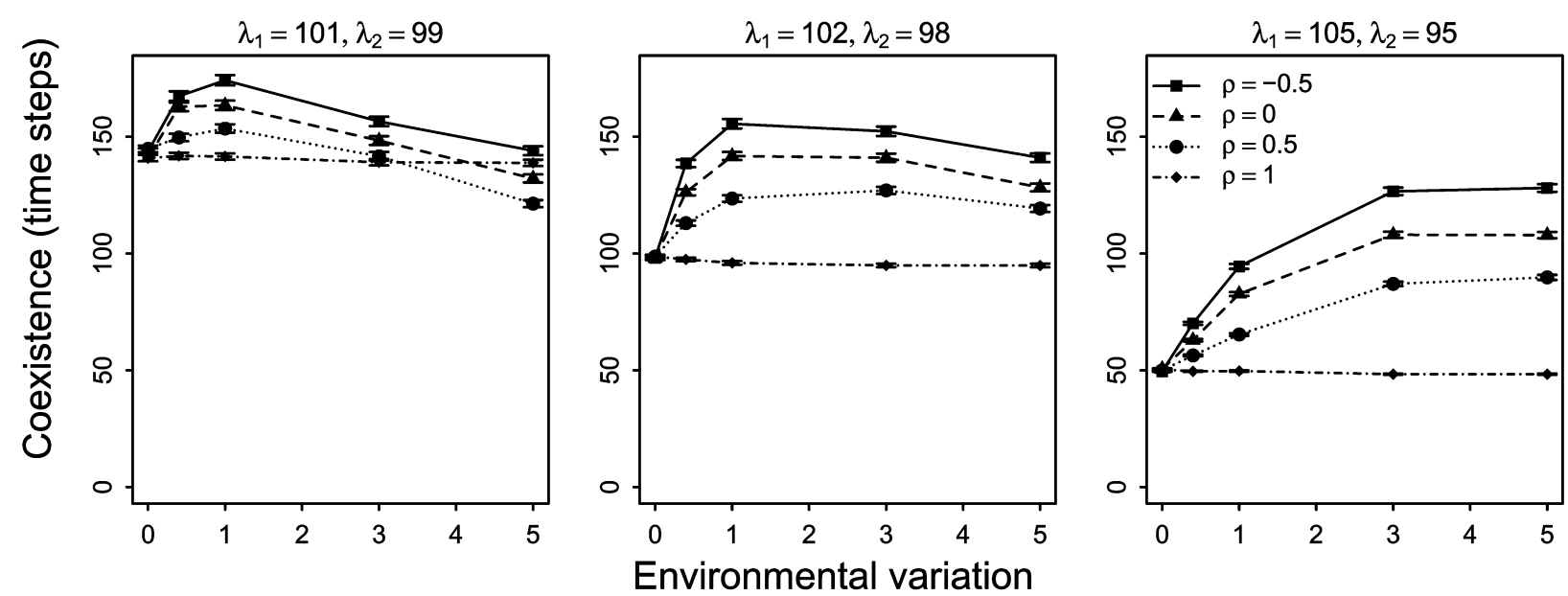

Figure 3: Relationship between environmental variation and the simulated mean time of coexistence under a range of parameter values. Competitive dominance (the difference in values of $\lambda$ ) increases from left to right. Lines and symbols represent different values of the correlation in species' responses to the environment, $\rho$. Bars show the standard error of the mean coexistence time.

For each run, we initialized the realized densities $\left(N_{i, 0}\right)$ of both populations at the equilibrium density of a onespecies model with $\lambda$ equal to the mean of $\lambda_{1}$ and $\lambda_{2}$ (in the absence of environmental variation) and then simulated until one species became extinct. We used a unique sequence of germination rates for each run, again simulating germination rates from a multivariate normal distribution. We recorded the time of extinction, the identity of the species becoming extinct, and the mean density of each species over the length of the run.

As a check on our conjecture that it is environmental variation that drives these patterns, we studied model sensitivity to initial population size, seed survival probability, and the absolute value of $\lambda$. We obtained similar results when we initialized the two species at low, high, or very unequal densities. Increasing the values of seed survival and $\lambda$ increased coexistence times but did not change the qualitative patterns.

\section{Results}

\section{Extinction and Stability}

In our analysis of a single-species system, the probability of extinction increased with increasing environmental variation (fig. 2A). Across a range of fecundities, the relationship between environmental variation and extinction risk was nonlinear, increasing slowly at very low levels of environmental variability and then increasing faster at moderate to high levels of variation.

In a two-species storage effect model without demographic stochasticity, the inferior competitor had negative low-density growth rates in a constant or nearly constant environment, implying certain extinction (fig. $2 B$ ). As environmental variation increased, however, the inferior competitor's low-density growth rate climbed above 0 , permitting long-term persistence. The shape of the relationship was concave down, with the most rapid increases in growth rates occurring at low levels of environmental variation. This result, which was robust to changes in the degree of competitive asymmetry (not shown), suggests diminishing returns of environmental variation on the inferior's average long-term growth rate.

\section{Coexistence Times}

Coexistence times in the simulated two-species system were short in the absence of environmental variation (fig. 3). As competitive asymmetry increased, coexistence time decreased further. When germination rates were perfectly correlated, $\rho=1$, meaning that species responded identically to the environment, increases in environmental variation led to slight decreases in coexistence time relative to the zero-variation case (fig. 3).

As long as species did not have identical germination rates, environmental variation almost always increased coexistence relative to the zero-variation case. Only when competitive dominance was very weak and environmental variation very strong did coexistence times drop below those of the zero-variation case (fig. 3, left). Decreases in the correlation in germination rates, $\rho$, increased coexistence times (fig. 3). The relationship between coexistence times and environmental variation was more complex. For a given level of competitive asymmetry, the longest coexistence times occurred at intermediate levels of vari- 

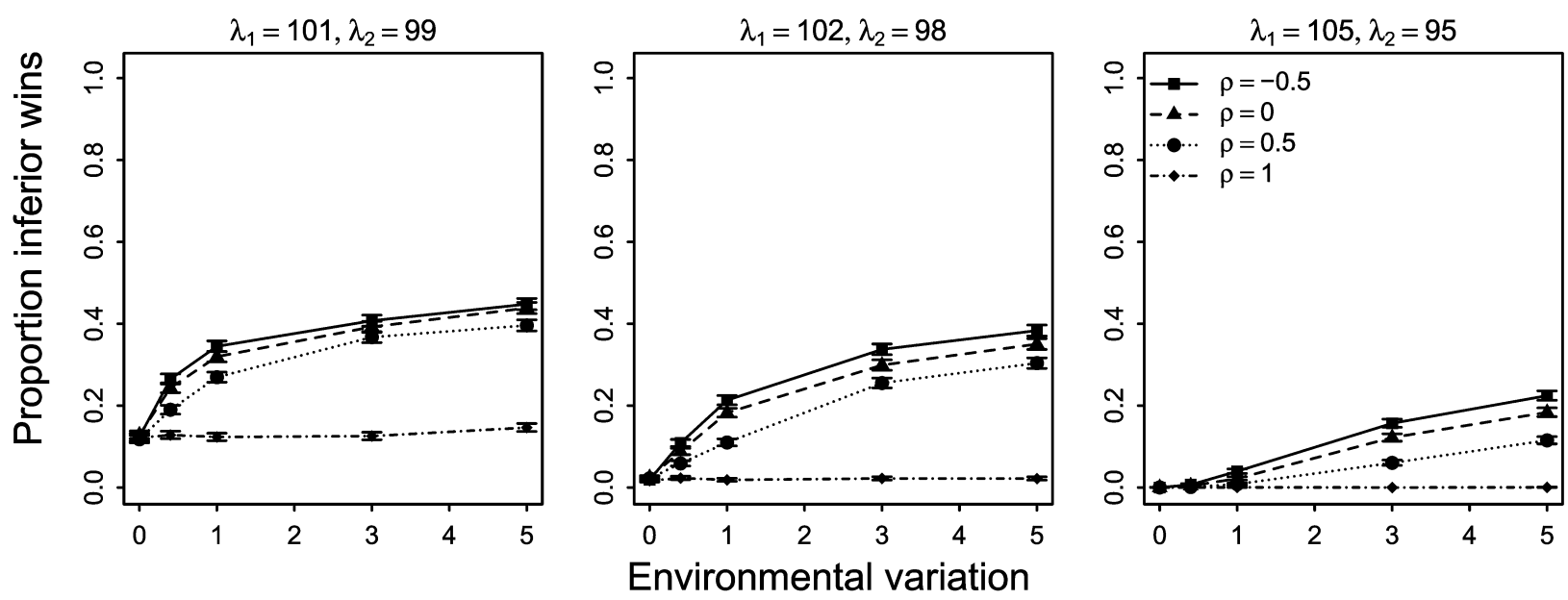

Figure 4: Fraction of runs in which the superior competitor became extinct before the inferior. Competitive dominance (the difference in values of $\lambda$ ) increases from left to right. Lines and symbols represent different values of the correlation in species' responses to the environment, $\rho$. Bars show $95 \%$ confidence intervals on the binomial parameter estimate.

ability, and the level of environmental variation producing the longest coexistence times increased with greater competitive asymmetry (fig. 3). The correlation in germination rates had a weaker influence on the optimal level of environmental variation: the peak in coexistence times occurred at slightly lower levels of variation with more negative values of $\rho$ (fig. 3, center). In summary, coexistence times were longest under low competitive dominance, negative correlations in species' response to the environment, and low to intermediate values of environmental variation.

\section{Extinction of Superior Species, Effects on Abundance, and the Distribution of Extinction Time}

In contrast to many competition models, in the storage effect model, it is possible for the inferior competitor to exclude the superior. In our simulations, the probability of the superior becoming extinct increased with environmental variation and decreased with competitive dominance and the correlation in species germination rates (fig. 4). Extinction of the superior species reached a maximum of $44 \%$ when competitive asymmetry was lowest and environmental variation greatest.

The mean density of the superior competitor increased with competitive dominance and the correlation in germination rates and decreased with environmental variation (fig. 5). For the inferior, mean density increased with environmental variation but decreased with the correlation in germination and with competitive dominance. All extinction times were right skewed (fig. 6). Skew was lowest when the correlation in germination rates was 1 (fig. 6, top row) and increased as correlation in germination de- creased and environmental variation increased. In most runs, one species became extinct quickly, but in a few runs, the two species coexisted for long periods (fig. 6).

\section{Discussion}

Our most important result is that the relationship between environmental variation and coexistence times in a twospecies storage effect model can have an intermediate maximum: as environmental variation increases, coexistence times first rise but then fall. Evidence that time to extinction may peak at an intermediate level of environmental variation contrasts with lessons from both singlespecies models, which have demonstrated a uniformly negative effect of environmental variation on persistence (Lewontin and Cohen 1969; Lande 1993; Alvarez 2001), and storage effect models, which imply a uniformly positive effect of variation on stable coexistence under certain conditions (Chesson and Warner 1981; Chesson and Huntly 1989). Our simulations support Hutchinson's (1961) intuition that the same environmental stochasticity that makes stable coexistence possible also carries the threat of extinction when combined with demographic stochasticity.

The relative strengths of stochastic extinction risk and competitive stabilization change across a gradient of environmental variation. In the single-species model that we used to demonstrate extinction risk (e.g., fig. $2 A$ ), every year is a good year in the absence of variation, meaning that extinction probability is 0 . As variation is increased, extinction risk increases slowly at first and then more rapidly (similar to fig. 2.3 in Morris and Doak 2003). In 

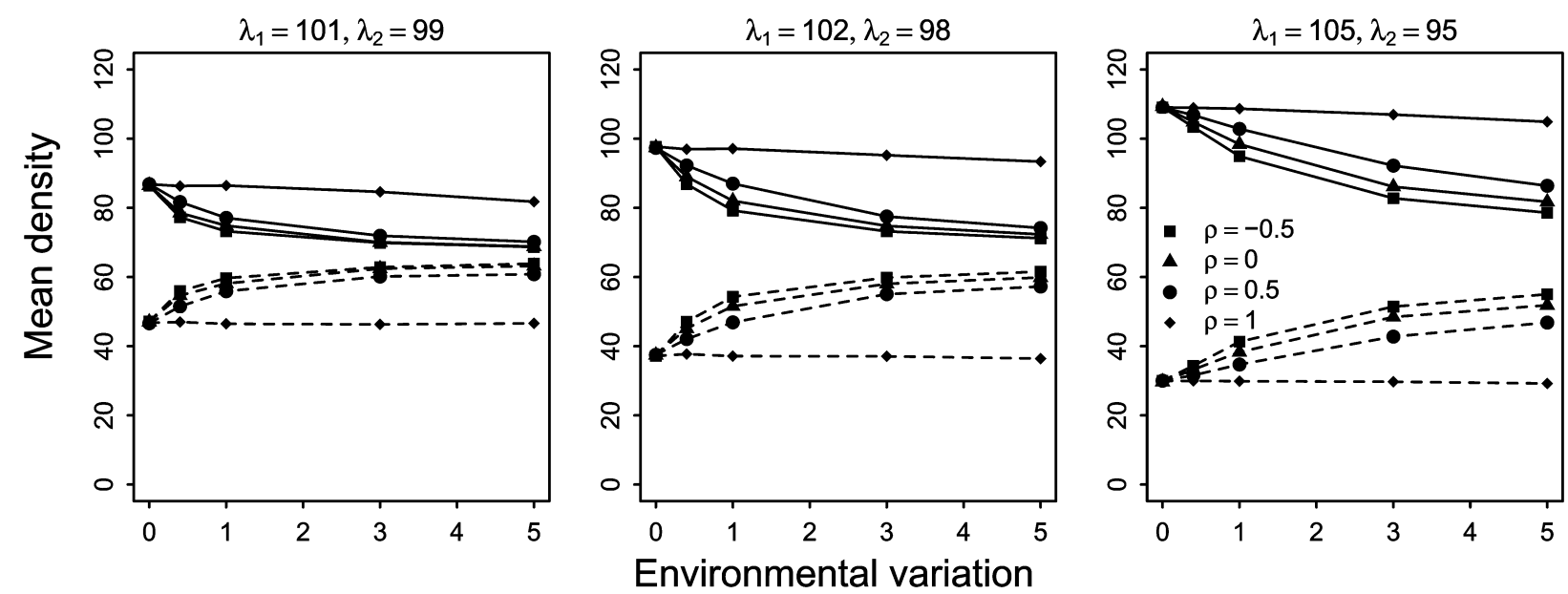

Figure 5: Mean density of the superior (solid lines) and inferior (dashed lines) competitors for various parameter combinations. Competitive dominance (the difference in values of $\lambda$ ) increases from left to right. Different symbols represent different values of the correlation in species' response to the environment, $\rho$.

contrast, for the inferior competitor in our two-species storage effect model, every year is a bad year in the absence of variability; introducing just a small amount of variability can make a good year possible (fig. $2 B$ ). As a result, the stabilizing influence of the storage effect accumulates rapidly at low levels of variation, when stochastic extinction risk is low, leading to longer coexistence times in our simulations. But as environmental variation increases further, long runs of bad years become more likely, and the probability of extinction due to demographic stochasticity increases, eventually overcoming the stabilizing effects of variability and decreasing coexistence times.

One surprising result was that at low levels of competitive dominance and high levels of environmental variation, differences in species' responses to the environment actually reduced coexistence times relative to the case in which species respond identically to the environment. The result is counterintuitive because differences in species' responses to the environment should help stabilize coexistence. The explanation may come from patterns of species density and extinctions of the superior competitor (figs. 4, 5). Under low competitive dominance and high environmental variation, both species are at risk of falling to low density and are therefore vulnerable to demographic stochasticity, whereas under higher competitive dominance or lower environmental variation, only the inferior species is vulnerable. This pattern is unchanged if we increase the mean fecundity $(\lambda)$ for the two species from 100 to 500 but keep the ratio $\lambda_{1} / \lambda_{2}$ the same (results not shown).

In principle, an alternative coexistence model could lead to different results if its stabilizing effects were weak at low levels of environmental variation. However, few coexistence mechanisms based on temporal environmental fluctuations have been discovered (Chesson 2000), and the storage effect is arguably the best developed. We speculate that an alternative model would have to be based on a significantly different mechanism in order to generate a monotonic relationship between environmental variation and coexistence times.

The optimal level of environmental variation for coexistence depends strongly on the degree of competitive dominance and more subtly on the correlation in germination rates. As competitive dominance increases, the amount of environmental variation that maximizes coexistence time also increases. This result is consistent with coexistence theory: as fitness inequality among species increases, stabilizing forces must become stronger (Chesson 2000; Adler et al. 2007). In the model analyzed here, the ratio $\lambda_{1} / \lambda_{2}$ is proportional to the fitness inequality, and environmental variation is an important component of stabilization. Similarly, as the correlation in germination rates increased, weakening stabilization, the level of environmental variation that optimized coexistence increased slightly.

The intermediate peak in the relationship between environmental variation and coexistence times complicates predictions about the effect of future increases in climate variability on population persistence. An increase in environmental variation could either increase or decrease a population's expected persistence time, depending on where the system is currently located on the variability gradient and how far it will shift. For example, if a population currently experiences less than the optimal level 

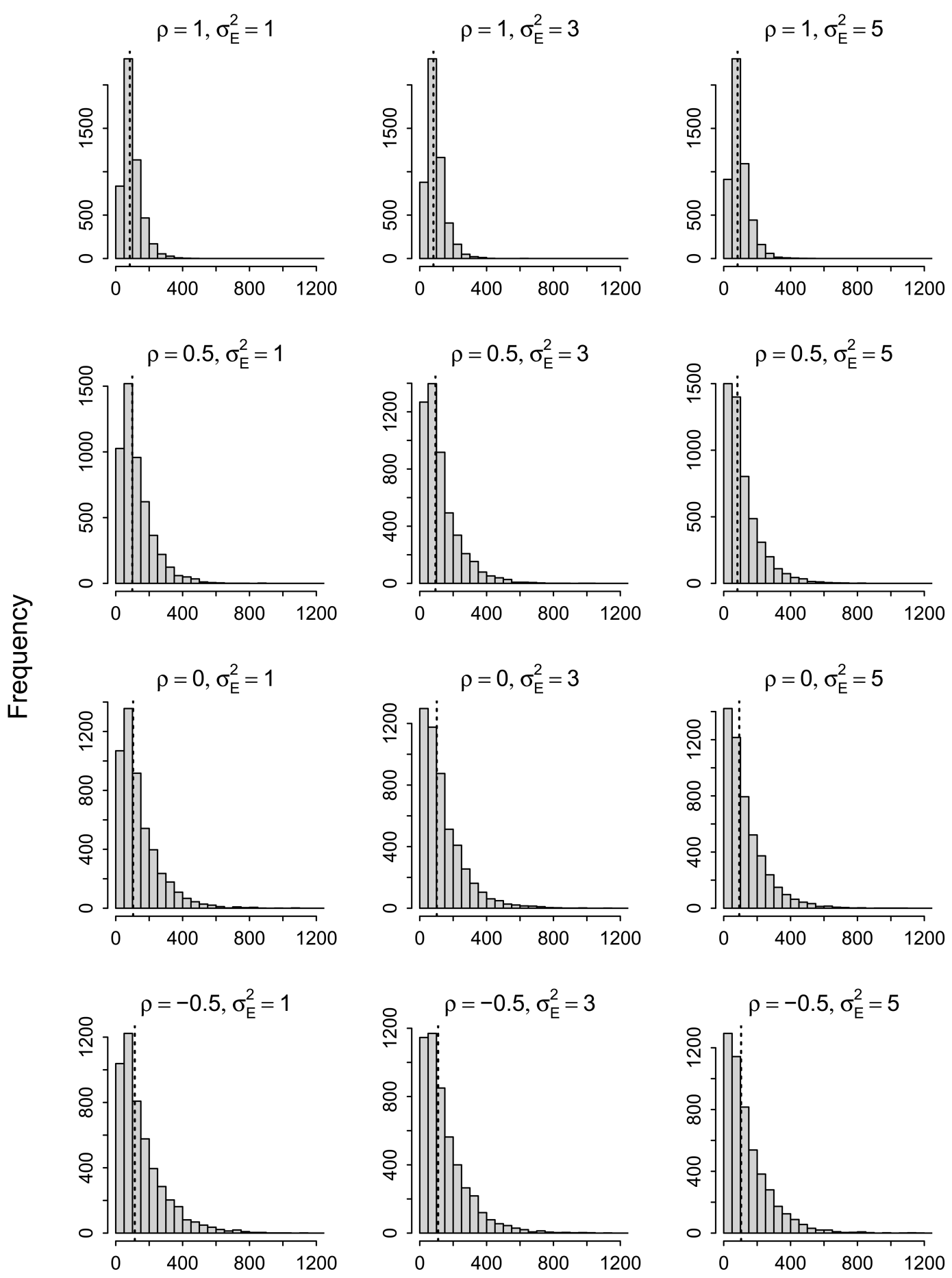

Time to first extinction

Figure 6: Distributions of coexistence times for different values of the correlation in germination rates $(\rho)$ and the environmental variance $\left(\sigma_{E}^{2}\right)$. For all runs, $\lambda_{1}=102$ and $\lambda_{2}=98$. Dashed lines indicate median coexistence times. 
of environmental variation, a small increase in variation might push it closer to the optimum, further stabilizing coexistence. However, a large increase in variation might move it well past the optimum, shortening the time to extinction.

Fortunately, our results provide some guidance in understanding where and when increases in environmental variation will increase or decrease coexistence times. First, environmental variation is most likely to increase persistence of species most vulnerable to competition. In our simulations, the superior competitor never benefited from variability. Furthermore, when competitive asymmetry is very strong, the optimal level of environmental variation for the inferior's persistence may be so high that it could never realistically be reached or that decreases in coexistence with increasing variation may be small (e.g., fig. 3, center). Second, because it is the combination of demographic and environmental stochasticity that causes extinction, our results apply to small populations. In large $\rightarrow$ populations, where the effect of demographic stochasticity is weak, even extreme environmental variation is unlikely to cause extinction of populations with seed banks or longlived adults. Third, we found that negative correlations in species' responses to the environment were associated with a relatively low optimal level of environmental variatior for coexistence, whereas positive correlations in species' responses corresponded to a higher optimal level of variation. Therefore, large increases in environmental variation are more likely to decrease coexistence times when species' responses to the environment are negatively correlated. Strong negative correlations in species' responses might reflect coevolution (Connell 1980; Thompsor $\rightarrow$ 1999), while weak or positive correlations would result from random species assembly and environmental sorting (Cavender-Bares et al. 2004; Ackerly and Cornwell 2007). The implication is that increases in environmental varia. tion would be more likely to destabilize coevolved systems than assemblages thrown together by chance.

Our findings highlight the role of demographic sto $\rightarrow$ chasticity in the extinction process and continue a recent trend of adding demographic stochasticity to classical coexistence models to gain new insight. For example, Tilman (2004) showed that demographic stochasticity creates limiting similarity in species assembly, producing more re alistic predictions about species abundance distributions than deterministic niche models. In our case, adding demographic stochasticity to a storage effect model reconciled contrasting predictions about the consequences of environmental variation for population persistence: environmental variation can increase the risk of stochastic extinction, as expected by single-species population theory at the same time that it has a stabilizing effect on coexistence, as expected by coexistence theory. This synthesis benefits both subdisciplines, helping conservation biologists anticipate when and where environmental variation might increase the persistence of an inferior competitor and helping coexistence theory make relevant predictions about the small populations that are the focus of conservation biology.

\section{Acknowledgments}

We thank J. Levine for many discussions that sharpened our thinking. Earlier versions of the manuscript were improved thanks to comments from H. Dalgleish, J. Levine, J. Mukherjee, L. Zachman, and two anonymous reviewers. P.B.A. was supported by National Science Foundation grant DEB-0614068.

\section{Literature Cited}

Ackerly, D., and W. Cornwell. 2007. A trait-based approach to community assembly: partitioning of species trait values into withinand among-community components. Ecology Letters 10:135-145.

Adler, P. B., J. HilleRisLambers, P. Kyriakidis, Q. Guan, and J. M. Levine. 2006. Climate variability has a stabilizing effect on coexistence of prairie grasses. Proceedings of the National Academy of Sciences of the USA 103:12793-12798.

$\rightarrow$ Adler, P. B., J. HilleRisLambers, and J. M. Levine. 2007. A niche for neutrality. Ecology Letters 10:95-104.

$\rightarrow$ Adondakis, S., and D. L. Venable. 2004. Dormancy and germination in a guild of Sonoran Desert annuals. Ecology 85:2582-2590.

Alvarez, L. 2001. Does increased stochasticity speed up extinction? Journal of Mathematical Biology 43:534-544.

Andrewartha, H. G., and L. C. Birch. 1954. The distribution and abundance of animals. University of Chicago Press, Chicago.

Bai, Y., X. Han, J. Wu, Z. Chen, and L. Li. 2004. Ecosystem stability and compensatory effects in the Inner Mongolia grassland. Nature 431:181-184.

Boyce, M. 1992. Population viability analysis. Annual Review of Ecology and Systematics 23:481-497.

Boyce, M., C. Haridas, C. Lee, C. Boggs, E. Bruna, T. Coulson, D. Doak, et al. 2006. Demography in an increasingly variable world. Trends in Ecology \& Evolution 21:141-148.

Cáceres, C. E. 1997. Temporal variation, dormancy, and coexistence: a field test of the storage effect. Proceedings of the National Academy of Sciences of the USA 94:9171-9175.

$\rightarrow$ Cavender-Bares, J., D. D. Ackerly, D. A. Baum, and F. A. Bazzaz. 2004. Phylogenetic overdispersion in Floridian oak communities. American Naturalist 163:823-843.

$\rightarrow$ Chesson, P. 1990. Geometry, heterogeneity and competition in variable environments. Philosophical Transactions of the Royal Society B: Biological Sciences 330:165-173.

$\rightarrow \quad$. 2000. Mechanisms of maintenance of species diversity. Annual Review of Ecology and Systematics 31:343-366.

Chesson, P., and N. Huntly. 1989. Short-term instabilities and longterm community dynamics. Trends in Ecology \& Evolution 4:293298.

Chesson, P. L., and R. R. Warner. 1981. Environmental variability promotes coexistence in lottery competitive environments. American Naturalist 117:923-943. 
$\rightarrow$ Cohen, D. 1966. Optimizing reproduction in a randomly varyin environment. Journal of Theoretical Biology 12:119-129.

$\rightarrow$ Connell, J. H. 1980. Diversity and the coevolution of competitors, or the ghost of competition past. Oikos 35:131-138.

$\rightarrow$ Drake, J. 2005. Population effects of increased climate variation. Proceedings of the Royal Society B: Biological Sciences 272:18231827.

$\rightarrow$ Facelli, J., P. Chesson, and N. Barnes. 2005. Differences in seed biology of annual plants in arid lands: a key ingredient of the storage effect. Ecology 86:2998-3006.

$\rightarrow$ Higgins, S. I., S. T. A. Pickett, and W. J. Bond. 2000. Predicting extinction risks for plants: environmental stochasticity can save declining populations. Trends in Ecology \& Evolution 15:516-520.

$\rightarrow$ Hutchinson, G. E. 1961. The paradox of the plankton. American Naturalist 95:137-145.

$\rightarrow$ Karl, T. R., and K. E. Trenberth. 2003. Modern global climate change Science 302:1719-1723.

$\rightarrow$ Lande, R. 1993. Risks of population extinction from demographir and environmental stochasticity and random catastrophes. American Naturalist 142:911-927.

$\rightarrow$ Levine, J. M., and M. Rees. 2004. Effects of temporal variability or rare plant persistence in annual systems. American Naturalist 164: 350-363.

$\rightarrow$ Lewontin, R., and D. Cohen. 1969. On population growth in a randomly varying environment. Proceedings of the National Academy of Sciences of the USA 62:1056-1060.
Menges, E. 2000. Population viability analyses in plants: challenges and opportunities. Trends in Ecology \& Evolution 15:51-56.

Morris, W. F., and D. F. Doak. 2003. Quantitative conservation biology: theory and practice of population viability analysis. Sinauer, Sunderland, MA.

Pake, C. E., and D. E. Venable. 1995. Is coexistence of Sonoran Desert annuals mediated by temporal variability in reproductive success? Ecology 76:246-261.

Salinger, M. 2005. Climate variability and change: past, present and future: an overview. Climatic Change 70:9-29.

$\rightarrow$ Thompson, J. 1999. The evolution of species interactions. Science 284:2116-2118.

Tilman, D. 2004. Niche trade-offs, neutrality, and community structure: a stochastic theory of resource competition, invasion, and community assembly. Proceedings of the National Academy of Sciences of the USA 101:10854-10861.

Tilman, D., and J. Downing. 1994. Biodiversity and stability in grasslands. Nature 367:363-365.

$\rightarrow$ Venable, D. L., and J. S. Brown. 1988. The selective interactions of dispersal, dormancy, and seed size as adaptations for reducing risk in variable environments. American Naturalist 131:360-384.

$\rightarrow$ Warner, R. R., and P. L. Chesson. 1985. Coexistence mediated by recruitment fluctuations: a field guide to the storage effect. American Naturalist 125:769-787.

Associate Editor: Andrew R. Solow Editor: Donald L. DeAngelis 